\title{
TESTES ESTATÍSTICOS E ANÁLISE MULTIVARIADA NA DETERMINAÇÃO DOS MODOS DE FALHA MONITORADOS PELOS INSTRUMENTOS EM BLOCOS DE CONCRETO DA BARRAGEM DE ITAIPU
}

\author{
O. C. SILVA ${ }^{1,2, *}$, J. M. MARQUES ${ }^{2}$ e I. P. NIERADKA ${ }^{1}$ \\ ${ }^{1}$ Universidade Estadual do Oeste do Paraná (UNIOESTE) \\ ${ }^{2}$ Universidade Federal do Paraná (UFPR) \\ orlandocs_santos@hotmail.com*
}

Artigo submetido em julho/2015 e aceito em agosto/2015

DOI: $10.15628 /$ holos. 2015.3168

\begin{abstract}
RESUMO
Monitorar uma barragem requer métodos tecnológicos que permitem uma avaliação mais precisa do comportamento de uma estrutura. Em uma estrutura de concreto que compõe uma barragem vários instrumentos devem ser instalados de tal maneira que permitam leituras que avalie se a estrutura está em boas condições, normalmente cada instrumento está destinado a coletar dados sobre características individuais da estrutura, possibilitando o diagnóstico de anomalias. O objetivo desse artigo é utilizar a instrumentação instalada em um bloco da barragem de
\end{abstract}

Itaipu, para determinar modos de falhas sem uma avaliação individual de cada instrumento. $O$ método baseia-se no trabalho apresentado em XXX SNGB Seminário Nacional de Grandes Barragens realizado em Foz do Iguaçu, que com o auxílio da análise fatorial, foi desenvolvido um método para determinação dos modos da falha possíveis de serem monitorados e de suas regiões críticas. Esse método será aplicado em um bloco chave de Itaipu. Pretende-se, com essa aplicação obter resultados que comprove a eficiência desse método.

PALAVRAS-CHAVE: segurança de barragens, análise fatorial, modos de falha.

\section{STATISTICAL TESTS ASSOCIATED WITH MULTIVARIATE ANALYSIS IN DETERMINING THE POSSIBLE WAYS OF FAULTS MONITORED BY INSTRUMENT IN CONCRETE BLOCKS OF ITAIPU DAM}

\begin{abstract}
Monitor a dam requires technological methods that allow a more accurate assessment of the behavior of a structure. In a concrete structure that makes up a dam various instruments must be installed in such a way to allow readings to assess whether the structure is in good condition, usually every instrument is designed to collect data on individual characteristics of the structure, enabling the diagnosis of abnormalities. The aim was to use the instrumentation installed in one of Itaipu dam block to determine failure modes without an individual
\end{abstract}

assessment of each instrument. The method is based on the work presented in XXX SNGB - National Seminar on Large Dams held in Foz do Iguaçu, which with the help of factor analysis, we developed a method for determination of possible failure modes to be monitored and their critical regions. This method will be applied in a Itaipu key block. It is intended, with that application results that prove the effectiveness of this method.

KEYWORDS: dam safety, factor analysis, failure modes. 


\section{INTRODUÇÃO}

A segurança das barragens constitui uma preocupação permanente para as entidades governamentais, tanto por sua importância econômica específica como pelo risco potencial que representa a possibilidade de ruptura ou outro acidente grave, em termos de vidas humanas, impacto ambiental, prejuízos materiais e os reflexos econômico-financeiros (ICOLD, 2003).

Segundo estimativas, no Brasil existem cerca de 13.529 barragens em cadastro no País, distribuídas pelos setores usuários ou produtivos da seguinte maneira: 11.748 usos múltiplos (87\%), 1.261 para geração de energia hidrelétrica (9\%), 264 de rejeitos de mineração (2\%) e 256 de resíduos industriais (2\%) (ANA, 2013).

Em 20 de setembro de 2010 entra em vigor (Brasil, 2010) que estabelece a Política Nacional de Segurança de Barragens destinadas à acumulação de água para quaisquer usos, à disposição final ou temporária de rejeitos e à acumulação de resíduos industriais, e cria o Sistema Nacional de Informações sobre Segurança de Barragens (PNSB).

Em seguida veio Brasil (2012) que estabelece diretrizes para implantação da política nacional de segurança de barragens, aplicação de seus instrumentos e atuação do sistema nacional de informação sobre segurança de barragens.

Diante de normas que visam o controle da segurança de barragens, neste artigo é proposta a aplicação de uma metodologia que é capaz de determinar modos de falha que são ocasionados por algum comportamento atípico da estrutura. Anomalias em uma estrutura nada mais são que movimentos não admissíveis, que podem ocasionar danos mais graves como riscos de acidentes (GUSMÃO FILHO, 2006).

Os instrumentos destinados a garantir a segurança de uma barragem, são capazes de identificar movimentos não admissíveis, fornecendo leituras que comprovem esse comportamento. O profissional em segurança de barragens é capaz de interpretar essas leituras como uma possível anomalia, e assim, promover alguma ação corretiva.

De acordo com Silveira (2003), Gusmão Filho (2006) e Eletrobrás (2003), alguns instrumentos são capazes de determinar uma mesma anomalia, o que indica que leituras fora de um determinado intervalo de confiança para esses instrumentos, apontam que tal anomalia pode estar ocorrendo, ou na eminencia de ocorrer.

A metodologia aplicada nesse artigo utiliza o conhecimento apresentado na literatura e a experiência dos profissionais em segurança de barragens de Itaipu, para determinar qual é a combinação de instrumentos que ocasionam, caso suas leituras estejam fora do que é normalmente é registrado, uma determinada anomalia, e após, determinar qual sua correspondente região de falha.

Para tanto, aplica-se uma técnica conhecida como Análise Fatorial, que possibilita o tratamento das leituras dos instrumentos como variáveis aleatórias. Assim, é possível gerar grupo de instrumentos que são altamente correlacionados, e desvendar relações entre eles que não são facilmente observáveis.

A aplicação da Análise Fatorial diminui o número de variáveis aleatórias envolvidas no problema e a determinação das regiões críticas de um grupo de anomalias auxilia o profissional em segurança de barragens na tomada de decisão. 


\section{CORRELAÇÃO DOS INSTRUMENTOS COM ANOMALIAS}

A barragem tem esforços de peso próprio e de água represada, que geram deformação e em consequência um movimento. Este movimento pode ser admissível ou não, conforme o tipo de barragem e se está bem projetada ou não (GUSMÃO FILHO, 2006).

Os problemas de movimento em uma barragem envolvem necessariamente os seguintes:

- Problemas de deformação, como recalque, tombamento, etc.;

- Problemas de permeabilidade, devido à percolação de água dentro dela ou na fundação, excesso da pressão hidrostática como poro pressões;

- Problemas de resistência ao cisalhamento, como o de escorregamento de taludes;

- Problemas de transbordamento, que gera forças não avaliadas em uma barragem.

Segundo Gusmão Filho (2006) a instrumentação de maciços rochosos visa determinar, fundamentalmente, as medições de deslocamentos, deformações, tensões, sub-pressões e vazões de drenagem, existentes na fundação de uma barragem. Os instrumentos mais usuais no monitoramento de barragens e que demonstram na prática melhor desempenho no campo são: os extensômetros múltiplos, pêndulos diretos e invertidos, piezômetros, inclinômetros e medidores de vazão.

A figura 1 apresenta uma correlação entre os tipos de instrumentos usualmente empregados na auscultação de barragens, conjuntamente com as inspeções visuais e os principais tipos de deterioração.

De acordo com a figura 1, muitos instrumentos têm a mesma finalidade, monitorando uma mesma anomalia. Assim, de acordo com esse quadro se um recalque diferencial estiver ocorrendo, o mesmo deverá ser detectado pelos pêndulos diretos, clinômetros, medidores de junta e os extensômetros. Caso ocorra uma distensão a montante, a percepção se dará através dos tensômetros, deformimetros, extensômetro e piezômetros. Essa propriedade apresentada pelos instrumentos é utilizada adiante para justificar o monitoramento através dos fatores, e dos escores fatoriais.

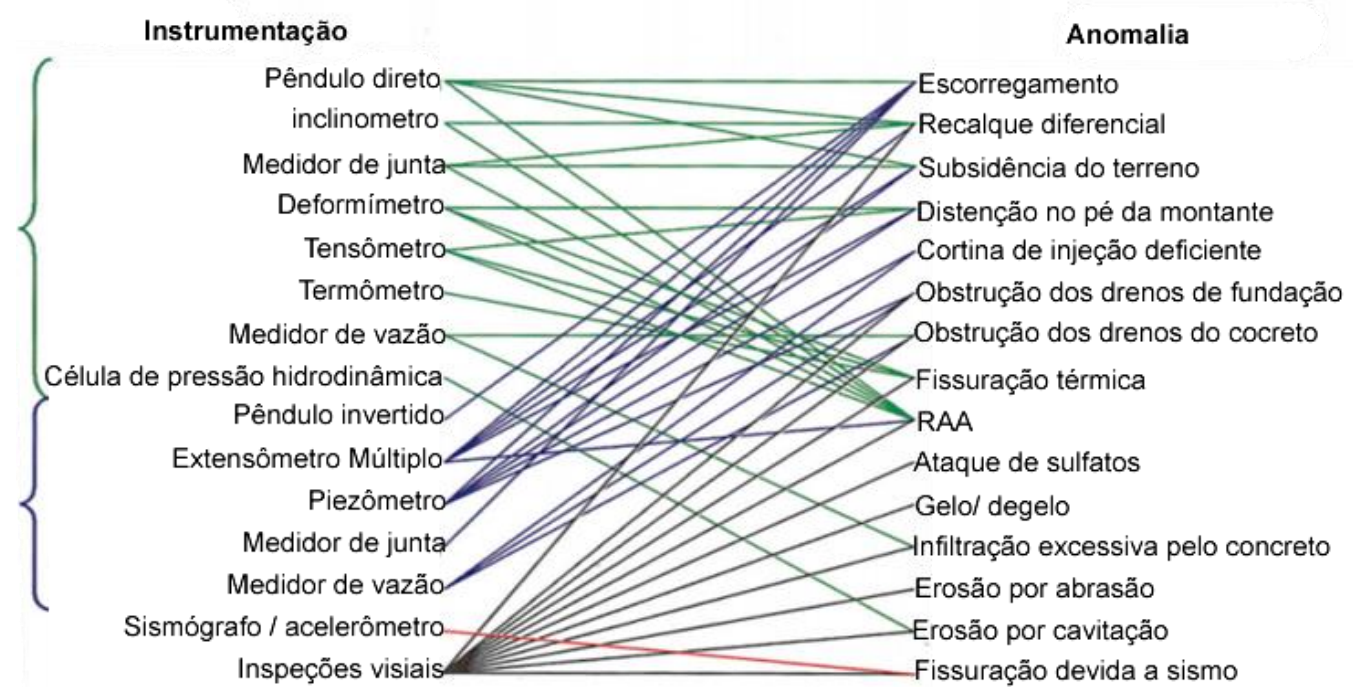

Figura 1: Instrumentação de barragens de concreto 


\section{DETERMINAÇÃO DOS MODOS DE FALHA}

A determinação dos modos de falha de uma estrutura de concreto altamente instrumentada foi um trabalho apresentado por Silva e Marques (2015), nesse trabalho o conceito de segurança de barragens foi discutido, apresentando os instrumentos mais utilizados no mundo todo para garantir a segurança de uma barragem. O trabalho foi inspirado em um bloco de concreto da usina hidrelétrica de Itaipu, e apresenta informações de como é realizado o monitoramento nas barragens.

Aplicando uma técnica da Análise Multivariada conhecida como Análise Fatorial, foi possível determinar grupos de instrumentos que são altamente correlacionados. Esses grupos são conhecidos como fatores e através deles é possível gerar os escores fatoriais, que são as variáveis aleatórias que substituíram as variáveis aleatórias iniciais (leituras dos instrumentos) simplificando a análise. Com o conhecimento adquirido após a análise fatorial e das correlações dos instrumentos com possíveis anomalias, desenvolveu-se um método capaz de determinar as regiões críticas de cada modo de falha.

Esse método consiste na realização de simulações nas leituras dos instrumentos de tal forma que fosse provocada cada anomalia, e para cada anomalia fosse realizada um grande número de simulações, gerando vários vetores com dados sintéticos que simulam a mesma anomalia. Com esses vetores é realizado o cálculo para geração dos escores fatoriais, uma técnica apresentada em diversos livros de Análise Multivariada tais como Johnson e Wichern (1998) e Corrar e Paulo Filho (2009).

Nesse contexto foi considerada como "falha na estrutura" uma mudança brusca em seu comportamento, não sendo necessariamente uma ação catastrófica, mas sim, um alerta de que algo de anormal está ocorrendo, e, que se não for reparado tal anomalia poderá ocorrer.

Silva e Marques (2015) utilizaram apenas os escores fatoriais que possuíam uma distribuição e probabilidade normal para aplicação do método. A exigência da condição de normalidade diminuía a eficiência do método, pois eliminavam da análise alguns fatores, onde possivelmente um conjunto importante de instrumentos estava associado, restringindo o número de anomalias possíveis de serem monitoradas.

Para o artigo proposto, é aplicado transformações nos dados, com o objetivo de transformar variáveis aleatórias com distribuição de probabilidade qualquer em variáveis aleatórias com distribuição de probabilidade normal. Para tanto é utilizado transformações sugeridas em Johnson e Wichern (1998) e que preserve mudança de comportamento.

O conceito de mudança de comportamento foi introduzido por Silva e Santos (2015) em um trabalho publicado em Congresso de Métodos Numéricos em Engenharia, realizado em Lisboa entre 29 de junho a 2 de julho. De acordo com o apresentado nesse artigo, não basta que uma função seja capaz de transformar um conjunto qualquer de dados em outro conjunto com distribuição normal de probabilidade, mas, que, além disso, ele preserve a mudança de comportamento dos dados. Isso impede erros no diagnóstico de algumas anomalias.

Considerando apenas os escores fatoriais que já possuem uma distribuição de probabilidade normal e aqueles onde foi possível encontrar uma função que transformasse os dados em normais, condição necessária para determinar as regiões críticas, é possível após 
identificar os instrumentos que estão associados aos fatores, gerar vetores de simulação que simulasse uma anomalia.

A próxima seção tem como objetivo resumir o procedimento utilizado para gerar o algoritmo apresentado por Silva e Marques (2015) para determinação das regiões críticas de cada modo de falha.

\section{DESCRIÇÃO DO MÉTODO}

Como análise fatorial agrupa os instrumentos altamente correlacionados a um mesmo fator, a ideia é utilizar as associações dos instrumentos com as anomalias apresentadas em Gusmão Filho (2006), Silveira (2003) e Eletrobrás (2003) e o conhecimento prático dos profissionais da Barragem de Itaipu, para relacionar os fatores com as anomalias.

Se mais de um fator for necessário para monitorar certa anomalia entramos no caso de modos de falha de sistemas, onde a ocorrência de mais de um evento é necessária para ocasionar uma falha.

Como não serão utilizadas as grandezas físicas, deve-se verificar o comportamento anormal da estrutura através das leituras dos instrumentos. De acordo com as literaturas citadas e do que foi repassado por profissionais que atuam há muitos anos em barragens, para que certas anomalias ocorram, é necessário que exista uma combinação de leituras fora do comportamento normal simultaneamente. Assim, deve-se determinar em quais fatores esses instrumentos estarão agrupados.

Como os escores fatoriais dependem dos fatores obtidos na análise fatorial e, consequentemente, dependem dos instrumentos agrupados neles, quando houver alguma mudança brusca nas leituras desses instrumentos correlacionados com uma determinada anomalia, o resultado do escore fatorial também deverá sofrer alteração. Portanto, para realizar esse monitoramento busca-se determinar as regiões críticas desses escores fatoriais a qual indicará quais as anomalias ocorreram.

Para determinar as regiões críticas são utilizados os intervalos de projeto dos instrumentos instalados no bloco e os intervalos de $95 \%$ de confiança da média dos últimos dois anos, e, determina-se um intervalo que representa uma mudança de comportamento do instrumento $k$, obtendo-se a Equação (1):

$M C_{k}=\left(\min \left\{l_{i}, L_{i}\right\}-k_{1} s, \operatorname{máx}\left\{l_{i}, L_{i}\right\}-k_{1} s\right) \cup\left(\min \left\{l_{s}, L_{s}\right\}+k_{2} s, \operatorname{má} x\left\{l_{s}, L_{s}\right\}+k_{2} s\right)$

onde, $l_{i}$ e $l_{s}$ são os limites inferiores e superiores do intervalo de confiança de $95 \%$ da média, respectivamente, $L_{i}$ e $L_{s}$ são os limites inferiores e superiores do intervalo de projeto, $s$ é o desvio padrão amostral das leituras do instrumento correspondente aos últimos dois anos, e, $\left\{k_{1}, k_{2}\right\}$ são constantes, maiores ou iguais a zero, que garantam que o intervalo de mudança de comportamento possa gerar valores extremos já obtidos pelas leituras de cada instrumento, mesmo que esses valores sejam raros de ocorrer.

Observe que o intervalo $M C_{k}$ assim definido apresenta valores entre os intervalos de projeto e o intervalo de confiança para média, e, ele não sugere valores impossíveis de serem alcançados, mas que não são normalmente registrados. Por esse motivo recebe o nome de 
intervalo de mudança de comportamento. Valores obtidos dentro desse intervalo não sugerem um problema eminente, já que podem estar dentro do intervalo de projeto ou de confiança, mas sugerem uma mudança no seu comportamento e podem servi como um aviso para um monitoramento mais detalhado.

Pode-se então definir o intervalo de bom comportamento do instrumento $k$, para os casos onde os instrumentos apresentam intervalos de projeto como mostra a equação 2.

$B C_{k}=\left(\operatorname{máx}\left\{l_{i}, L_{i}\right\}-k_{1} s, \min \left\{l_{s}, L_{s}\right\}+k_{2} s\right)$

onde $l_{i}$ e $l_{s}$ são os limites inferiores e superiores do intervalo de confiança de $95 \%$ da média, respectivamente, $L_{i}$ e $L_{s}$ são os limites inferiores e superiores do intervalo de projeto, respectivamente, $s$ é o desvio padrão amostral das leituras do instrumento correspondente aos últimos dois anos, e, $\left\{k_{1}, k_{2}\right\}$ são as mesmas constantes que formaram o intervalo de mudança de comportamento do instrumento $k$ correspondente.

Não existe uma regra geral para a determinação dos valores de $\left\{k_{1}, k_{2}\right\}$, pois os mesmos dependem da característica de cada instrumento, de sua sazonalidade e principalmente do nível de sensibilidade que o proprietário da barragem desejar. Por exemplo, valores próximos de zero para essas constantes, fornecem uma sensibilidade maior, pois incluem valores já ocorridos nos últimos anos como uma mudança no comportamento. Já valores maiores podem não acusar um comportamento já ocorrido como anormal, mesmo que essa leitura tenha sido muito rara e mereça atenção.

Usando um programa gerador de números aleatórios, já implementado no software Matlab é possível gerar vetores de simulação das anomalias, que são possíveis de monitorar a partir do que foi apresentado pela análise fatorial. Por exemplo, se três instrumentos estão relacionados com certa anomalia, basta gerar leituras para esses instrumentos em seus respectivos intervalos $M C$, e para os demais instrumentos gerar leituras dentro dos intervalos $B C$ obtidos através do intervalo de confiança de $95 \%$ da média $I C$, fazendo isso, temos a geração de um vetor que simula essa anomalia.

É importante destacar, que essa geração por mais que seja aleatória deve seguir o seguinte princípio, se existem três instrumentos altamente relacionados com essa anomalia, então antes é necessário verificar qual é o instrumento com maior influência no fator, essa informação é revelada pela análise fatorial. Além disso, a análise fatorial também revela qual é a correlação entre todos os instrumentos nesse estudo.

Assim, reordenamos os instrumentos, de forma que todos aqueles relacionados com a anomalia fiquem nos primeiros lugares, e, o primeiro instrumento deve ser o que possui maior influência no fator. Dessa forma, quando for gerado o primeiro número aleatório para o primeiro instrumento, é verificado em qual dos conjuntos do intervalo $M C$ ele pertence, visto que o intervalo $M C$ é formado pela união de dois conjuntos. Se por exemplo, for gerado um número do intervalo esquerdo do conjunto $M C$, quando for gerado o próximo número aleatório para o próximo instrumento, deve ser verificado qual é a correlação do segundo instrumento com o primeiro. Se a correlação for positiva, este número aleatório deve estar do mesmo lado esquerdo do intervalo $M C$ do segundo instrumento, caso contrário, ou seja, se a correlação for negativa, o número aleatório deve estar do lado direito do intervalo $M C$ do segundo instrumento. Seguindo esse princípio para todos os instrumentos, mesmo aqueles que não têm relação com a anomalia, 
contudo, neste caso, deve ser verificado o lado esquerdo e/ou direito do intervalo de bom comportamento $B C$ de tais instrumentos, visto que, todos intervalos contém a média das leituras e podem ser escritos como a união de dois conjuntos, ou seja, $B C=\left(b_{i}, \bar{x}\right) \cup\left(\bar{x}, b_{s}\right)$, onde $b_{i}$ e $b_{s}$ são os limites inferiores e superiores do respectivo intervalo de bom comportamento e $\bar{x}$ é a média amostral dos últimos dois anos dos respectivos instrumentos.

Esse processo tem a finalidade de respeitar o comportamento da estrutura, onde os instrumentos são altamente correlacionados. Dessa forma, é garantido um vetor de simulação condizente com a realidade. $O$ algoritmo de geração do vetor de simulação e obtenção da região crítica para uma determinada anomalia é descrito como segue.

Considere certa anomalia $i$, e suponha que existam $m$ instrumentos associados com a anomalia $i$. Considere também que desses $m$ instrumentos um número $m^{\prime}<m$ esteja altamente correlacionado com um fator $j$.

Passos:

10. Ordene os $m$ instrumentos em ordem decrescente, isto é, de forma que o instrumento com maior influência no fator esteja em primeiro lugar no vetor de simulação e sucessivamente;

2‥ Gere um número aleatório para o primeiro instrumento no seu respectivo intervalo $M C$;

3‥ Gere números aleatórios para os instrumentos $2, \cdots, m^{\prime}$, dos seus respectivos intervalos $M C$, verificando suas correlações com o primeiro instrumento;

4․ Gere os demais números aleatórios para os demais instrumentos, dentro dos seus respectivos intervalos de bom comportamento $B C$, verificando as correlações com o primeiro instrumento;

50. Gerar o vetor de simulação, padronizando todas as entradas, subtraindo as respectivas médias, e dividindo pelos respectivos desvios padrões de cada instrumento;

6‥ Multiplique esse vetor pelo vetor de coeficientes fatoriais do fator $j$, some os resultados para obter o valor do escore fatorial correspondente $E_{i j}$;

70. Padronize $E_{i j}$ obtendo $z_{i j}$, se necessário aplique antes a transformação em $E_{i j}$ para torná-lo uma variável aleatória normal;

8‥ Plote $z_{i j}$ no eixo $x$, abaixo da curva normal padrão;

9‥ Repita esse processo para um número suficientemente grande de vezes.

Nesse algoritmo, $E_{i j}$ representa o valor encontrado do escore fatorial $j$ quando simulado a anomalia $i, z_{i j}$ é a transformação desse escore em variável normal padrão.

Como o escore fatorial $j$ é uma variável aleatória normal, e o valor $z_{i j}$ foi obtido simulando uma situação atípica, o valor obtido para ele se distanciará da média 0 . Se for realizada essa simulação para um número suficientemente grande de vezes, então é obtido um número suficientemente grande de pontos no eixo $x$, que normalmente distanciarão da média, formando uma região em uma ou ambas as caldas da curva de Gauus, assim é utilizado essas regiões para determinar a região crítica da anomalia $i$, monitorada a partir do fator $j$. A figura 2 adiante apresenta um fluxograma desse algoritmo. 
Para determinar as regiões críticas, são analisados os pontos plotados no eixo $x$. Escolhemos a região onde eles se agrupem, ignorando um número de pontos fora dela, dependendo do percentual de acerto que se deseja. Se um percentual de $90 \%$ for satisfatório ao simular um número suficientemente grande de vetores, pode-se rejeitar $10 \%$ dos pontos que estejam distantes da região onde eles mais se agrupem, e para essa região, determinar um intervalo, considerando ele como um intervalo da reta real, mesmo sabendo que esse conjunto possui pontos discretos de simulações, porém, em $90 \%$ das vezes em que foi simulada aquela anomalia, os pontos se acumularam nesse intervalo.

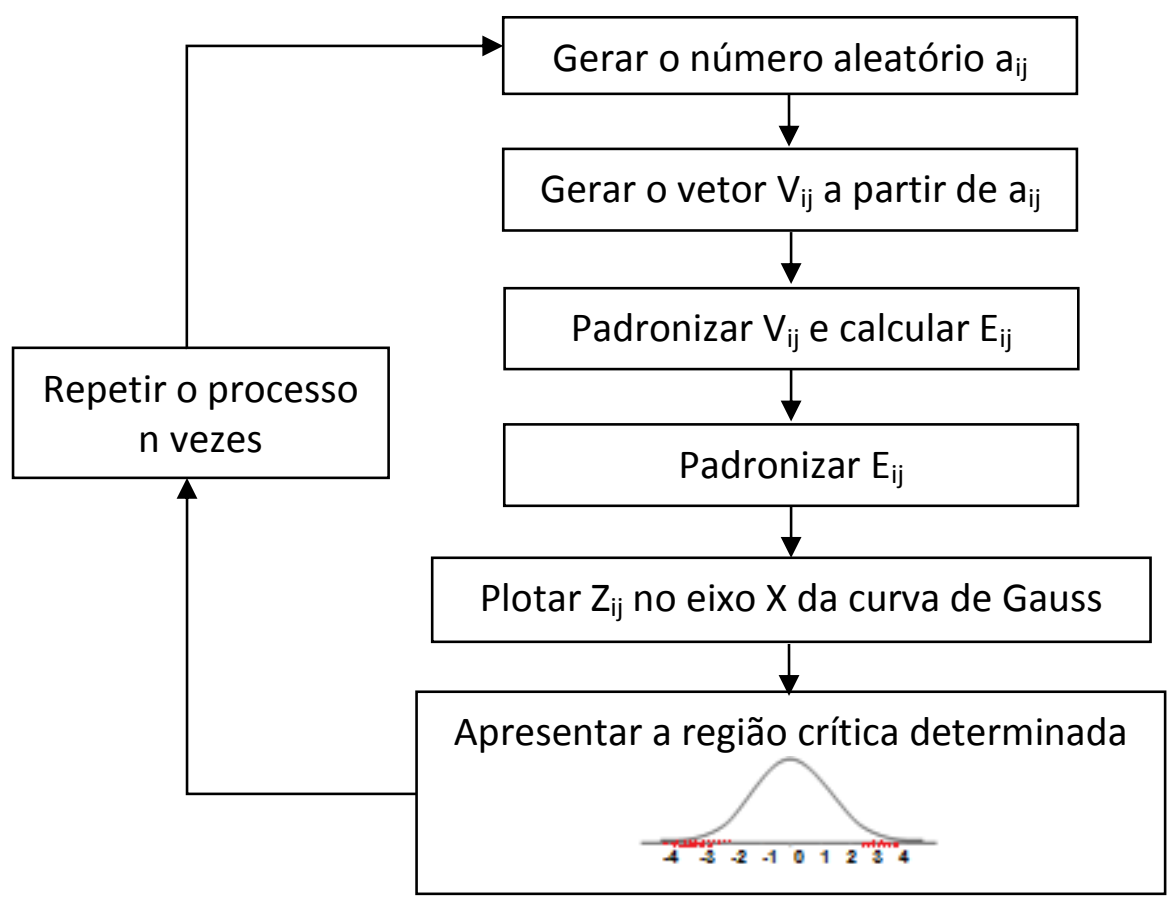

Figura 2: Fluxograma do algoritmo para determinar os modos de falha

Suponha que a figura 3(a), adiante, mostra a simulação de uma certa anomalia, nesse caso os pontos se concentram na calda direita da curva de Gauss, com a ocorrência de poucos pontos próximos da média. Nesse caso ignoramos esses pontos próximos da média e determinamos a região crítica como sendo o intervalo formado por dois pontos de simulação de tal forma que a região onde os pontos mais se agruparam pertençam a esse intervalo.

A figura $3(b)$, adiante, o caso foi oposto ao anterior, pois o pontos se concentraram na calda esquerda da curva de Gauss, e, de forma análoga defini-se um intervalo da reta formada por dois pontos de simulação, de tal forma que a região onde os pontos se concentraram esteja contida nesse intervalo.

A figura 3(c), adiante, apresenta o caso onde uma anomalia foi simulada e houve concentração dos pontos nas duas caldas da curva de Gauss. Nesse caso rejeitamos um número pequeno de pontos próximos à média, e assumimos como região crítica a união dos intervalos que contenham as regiões onde os pontos mais se concentraram nas duas caldas da curva de Gauss.

Assim, coletadas as leituras dos instrumentos instalados em algum bloco chave de uma barragem de concreto, feita as transformações necessárias de tal forma que cada instrumento possua apenas um representante mensal, e, calculado os escores fatoriais que sejam capazes de 
monitorar as anomalias, pode-se verificar se o valor desses escores fatoriais pertencem à uma determinada região crítica dessas anomalias. Caso ocorra, é dito que existem indícios estatísticos para acreditar que essa anomalia pode estar ocorrendo.
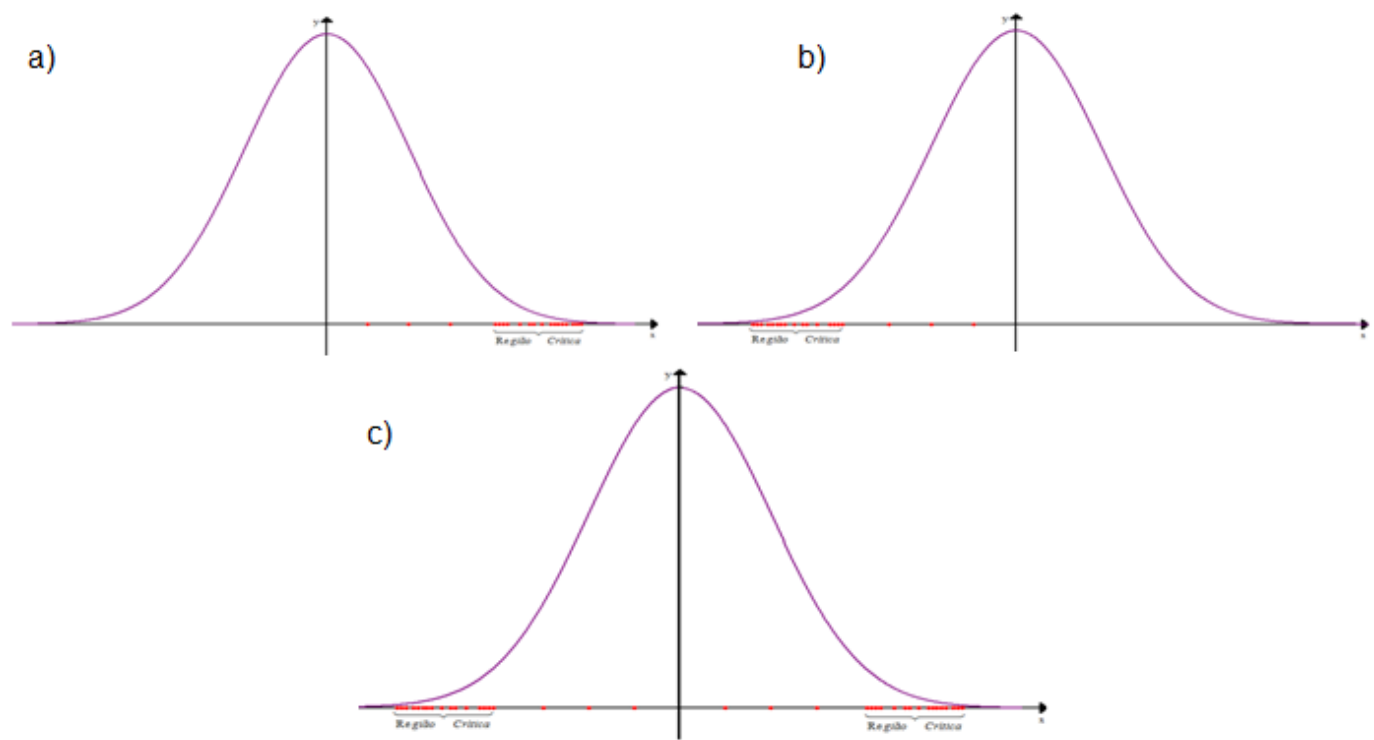

Figura 3: Representação das regiões críticas

\section{APLICAÇÃO DO MÉTODO PARA O BLOCO CHAVE DE ITAIPU}

Nesta seção aplica-se o método descrito anteriormente em um bloco chave da barragem de Itaipu. Os materiais empregados nesse estudo são as variáveis aleatórias geradas pelas leituras dos instrumentos instalados no bloco chave A-15, bloco do vertedouro. No total foram 50 instrumentos analisados, a grande maioria instalada apenas no bloco $A-15$, porém alguns instrumentos também pertencem aos blocos D-1 e A-14, pois estão instalados na divisa desses blocos para monitorar aberturas, recalques e deslizamentos. A figura 4 apresenta o bloco de Itaipu selecionado.

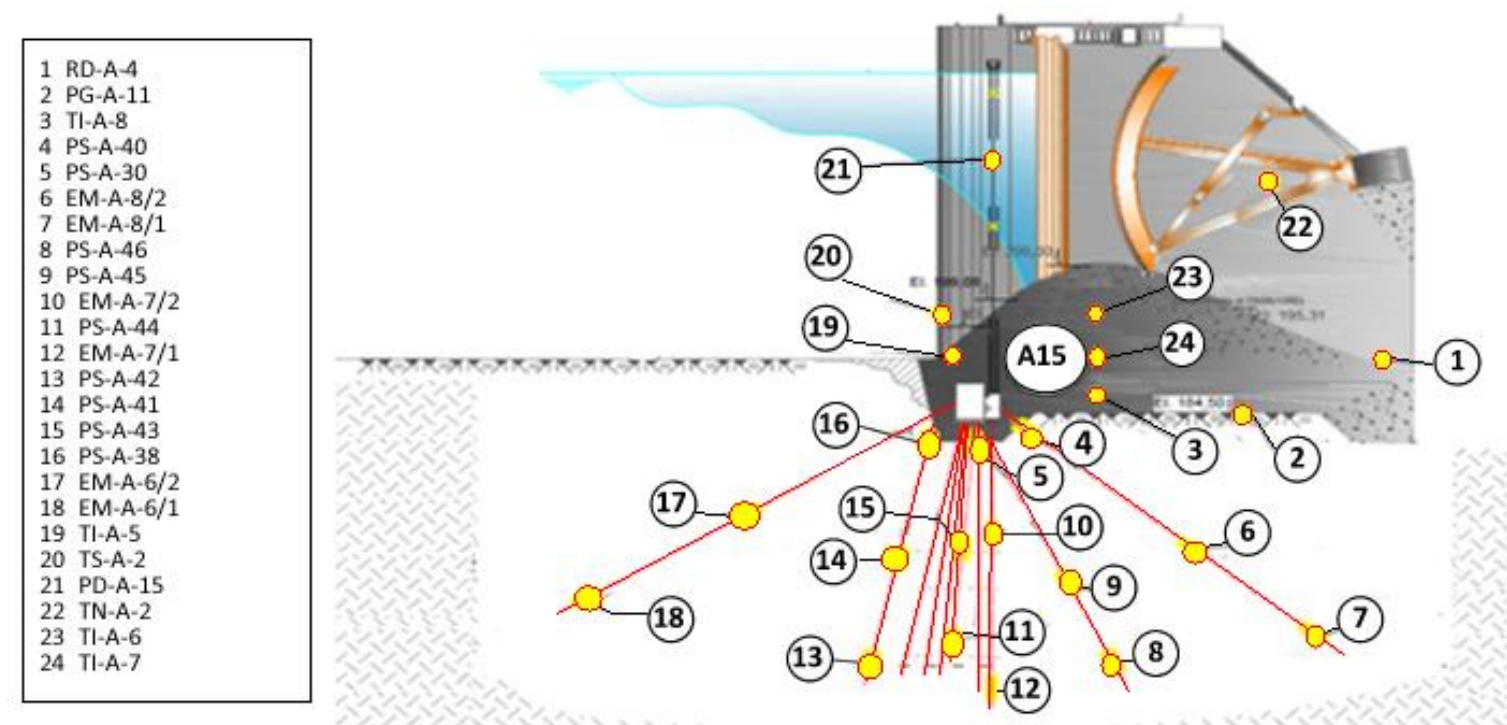

Figura 4: Bloco A-15 da barragem de Itaipu 
Os instrumentos instalados na barragem de Itaipu e as funções que desempenham na estrutura são encontrados em Itaipu (2003), pode ser visto no quadro 1 uma breve descrição dos instrumentos que são utilizados nesse estudo, apenas aqueles que estão instalados nos blocos de interesse. O quadro 2 apresenta a descrição de funcionamento dos instrumentos instalados no bloco A-15 e estão disponíveis nos manuais Sistema de Operação e Manutenção de Itaipu (SOM) que foram fornecidos pela equipe técnica de Itaipu.

É criada uma matriz $M_{168 \times 50}$ multivariada, com as leituras geradas pelos instrumentos apresentados no quadro 1 acrescentados de mais dois instrumentos que não estão instalados no bloco A-15 e nem nos blocos de divisa, tratam do termômetro que mede a temperatura ambiente e do instrumento que mede o nível do reservatório, totalizando 50 instrumentos em análise, esses instrumentos foram acrescentados por influenciar todos os blocos da Barragem de Itaipu. Foi considerado um período de tempo a partir de janeiro de 2000 até dezembro de 2013. As leituras no geral não estavam padronizadas, então para possibilitar a análise, os dados foram padronizados mensalmente. Para os instrumentos que possuíam mais de uma leitura mensal, foram calculadas as médias, que é o caso da maioria. No entanto, alguns instrumentos possuíam apenas leituras trimestrais, o caso dos Termômetros ( $\mathrm{TI}$ ), neles foi aplicado a técnica de interpolação Spline Cúbica, implementada no programa matemático Matlab.

Quadro 1: Instrumentos instalados no bloco A-15 do vertedouro

\begin{tabular}{|c|c|c|c|}
\hline \multicolumn{4}{|c|}{ INSTRUMENTOS BLOCO A-15 } \\
\hline INSTRUMENTOS & QUANTIDADES & UNIDADE DE LEITURA & FUNÇÃO \\
\hline Pêndulo (PD) & 1 & $\mathrm{~mm}$ & $\begin{array}{l}\text { Mede os deslocamentos na direção da } \\
\text { jusante e de montante. }\end{array}$ \\
\hline Extensômetro (EM) & 6 & $\mathrm{~mm}$ & $\begin{array}{l}\text { Mede o deslocamento e deformações que } \\
\text { ocorrerão entre pontos no maciço da } \\
\text { fundação. }\end{array}$ \\
\hline $\begin{array}{l}\text { Base de Alongâmetro } \\
\text { (JS) }\end{array}$ & 10 & $\mathrm{~mm}$ & $\begin{array}{l}\text { Medidor superficial de Junta para o } \\
\text { Concreto. }\end{array}$ \\
\hline Piezômetro Elétrico (PG) & 1 & $\mathrm{msnm}^{*}$ & $\begin{array}{l}\text { Mede a Subpressão dos poros no núcleo e } \\
\text { no contato núcleo-fundação. }\end{array}$ \\
\hline $\begin{array}{l}\text { Tensômetro de } \\
\text { Concreto (TN) }\end{array}$ & 1 & $\mathrm{Kgf} / \mathrm{cm}^{2}$ & $\begin{array}{l}\text { Para medição direta das tensões de } \\
\text { compressão no concreto. }\end{array}$ \\
\hline $\begin{array}{l}\text { Termômetro Superficial } \\
\text { (TS) }\end{array}$ & 1 & ${ }^{\circ} \mathrm{C}$ & $\begin{array}{l}\text { Termômetro embutidos no concreto para } \\
\text { medição da temperatura durante a } \\
\text { construçãoe operação. }\end{array}$ \\
\hline $\begin{array}{l}\text { Piezômetro Standpipe } \\
\text { (PS) }\end{array}$ & 9 & $\mathrm{msnm}^{*}$ & $\begin{array}{l}\text { Piezômetros para medir a pressão dos } \\
\text { poros e a subpressão hidráulica em juntas } \\
\text { ou contatos escolhidos no maciço rochoso } \\
\text { ou no contato barragem-fundação }\end{array}$ \\
\hline Termômetro Interno (TI) & 4 & ${ }^{\circ} \mathrm{C}$ & $\begin{array}{l}\text { Termômetros embutidos no concreto para } \\
\text { medição da temperatura durante a } \\
\text { construção e operação. }\end{array}$ \\
\hline Roseta Deformação (RD) & 15 & $\mathrm{Kgf} / \mathrm{cm}^{2}$ & $\begin{array}{l}\text { Embutidas no concreto para medição das } \\
\text { variações de volume autógenas, fluência e } \\
\text { deformação elástica e temperatura. }\end{array}$ \\
\hline
\end{tabular}


Quadro 2: Descrição de Funcionamento dos Instrumentos

\begin{tabular}{|c|c|c|c|c|}
\hline Sigla & Instrumentos & Faixa de leitura & Precisão & Sensibilidade \\
\hline TS & Termômetro Superficial & $-30^{\circ} \mathrm{C}$ à $70^{\circ} \mathrm{C}$ & $\pm 0,3^{\circ} \mathrm{C}$ & $0,1^{\circ} \mathrm{C}$ \\
\hline PG & Piezômetro Elétrico & $0-10 \mathrm{kgf} / \mathrm{cm}^{2}$ & $0,5 \%$ & $0,03 \mathrm{kgf} / \mathrm{cm}^{2}$ \\
\hline $\mathrm{TN}$ & Tensômetro & $\mathrm{C}-1500: 0-100 \mathrm{Kgf} / \mathrm{cm}^{2}$ & $\leq 0,01 \%$ & $0,01 \mathrm{ohm}$ \\
\hline $\mathrm{TI}$ & Termômetro Interno & $-30^{\circ} \mathrm{C}$ à $70^{\circ} \mathrm{C}$ & $\pm 0,3{ }^{\circ} \mathrm{C}$ & $0,1^{\circ} \mathrm{C}$ \\
\hline $\mathrm{EM}$ & Extensômetro & 0 à $50 \mathrm{~mm}$ & $\pm 0,01 \mathrm{~mm}$ & $0,01 \mathrm{~mm}$ \\
\hline \multirow{2}{*}{ OS } & $\begin{array}{c}\text { Piezômetro Standpipe } \\
\text { sem manômetro }\end{array}$ & $\begin{array}{c}\text { cota piezométrica entre a } \\
\text { cota de instalação e cota da } \\
\text { boca do tubo de leitura }\end{array}$ & $\pm 30,0 \mathrm{~mm}$ & $3,0 \mathrm{~mm}$ \\
\cline { 2 - 5 } & $\begin{array}{c}\text { Piezômetro Standpipe } \\
\text { com manômetro }\end{array}$ & $\begin{array}{c}\text { cota do manômetro mais o } \\
\text { valor do fundo de escala }\end{array}$ & $\begin{array}{c}5 \% \text { fundo de } \\
\text { escala }\end{array}$ & $\begin{array}{c}\text { depende do manômetro } \\
\text { instalado }\end{array}$ \\
\hline RD & Roseta de Deformímetro & $\begin{array}{c}1500 \times 10^{-6} \mathrm{~mm} \text { em ambos } \\
\text { sentidos }\end{array}$ & $\leq 0,01 \%$ & 0,01 ohm \\
\hline PD & Pêndulo Direto & $150 \mathrm{~mm}$ à $300 \mathrm{~mm}$ & $\pm 0,2 \mathrm{~mm}$ & $0,1 \mathrm{~mm}$ \\
\hline JS & Base de Alongâmetro & $397,5 \mathrm{~mm}$ à $402,5 \mathrm{~mm}$ & $\pm 0,005 \mathrm{~mm}$ & $0,001 \mathrm{~mm}$ \\
\hline
\end{tabular}

\section{TESTES ESTATÍSTICOS}

Antes de aplicar a análise fatorial na matriz $M_{168 \times 50}$ utilizando o Software Statistica, verificou-se alguns testes estatísticos, indispensáveis para aplicação do método.

Inicialmente foi testada a normalidade multivariada da matriz $M_{168 \times 50}$. Foi utilizado o teste computacionalmente intensivo baseado na distância de Mahalanobis. A hipótese nula desse teste afirma (BIASE, FERREIRA, 2012):

$H_{0}$ : A matriz de dados amostrais é proveniente de uma população normal multivariada.

A aplicação do teste, programado no Matlab, resultou em $p=0,7626$ (obtido com 100.000 simulações Monte Carlo). Portanto, ao nível de significância de 0,05, a hipótese de normalidade multivariada não pode ser descartada.

Sabendo-se da normalidade multivariada dos dados, aplicou-se o Teste de Esfericidade de Bartlett para testar as hipóteses:

$H_{0}$ : A matriz de correlação é igual a matriz identidade, ou seja, $\rho=\mathrm{l}$;

$H_{1}$ : A matriz de correlação é diferente da matriz identidade, ou seja, $\rho \neq \mathrm{I}$.

A aplicação do teste resultou em $\chi^{2}=7988,4, p=0$, ou seja, rejeitou-se a hipótese $H_{0}$. Portanto, a amostra multivariada é proveniente de uma população com matriz de correlação significativamente diferente da matriz identidade.

O cálculo da medida de adequacidade dos dados para realização de uma análise fatorial resultou em $K M O=0,8348$. Como o valor mínimo exigido é 0,50, a amostra multivariada satisfaz essa condição.

\subsection{Extrações dos Fatores}

Comprovados os resultados dos testes, aplicou-se a análise fatorial na matriz $M_{168 \times 51}$. O método adotado para obtenção dos coeficientes fatoriais foi o Método das Componentes Principais com rotação Varimax, visto que esse método obteve uma maior porção da variância explicada, comparado com o método da Máxima Verossimilhança. 
Aplicando o Critério de Kaiser do mínimo autovalor, foi obtido 9 fatores, que explicam $86,21 \%$ de toda variância, entretanto, duas variáveis apresentaram comunalidades baixas, menores que 0,70 , e devido a isso uma nova análise foi realizada removendo essas variáveis. A nova análise envolveu todos os testes estatísticos realizados anteriormente, mas agora em uma matriz $M_{168 \times 48}$ para reconfirmar adequação dos dados. Novamente todos os testes foram bem sucedidos.

Com a nova análise obteve-se uma variância explicada de $87,30 \%$, sem nenhuma variável com baixa comunalidade.

Os escores fatoriais gerados a partir dos coeficientes fatoriais são utilizados para a determinação dos modos de falha. Neles é aplicado o teste de normalidade univariada conhecido como Teste de Lilliefors, para comprovar se são variáveis aleatórias com distribuição de probabilidade normais, caso contrário, será necessária à aplicação de transformações nos escores. Aquelas variáveis que passarem nos testes serão padronizados e utilizados nas simulações das anomalias.

O Teste de Lilliefors (LF) é uma modificação do teste de Kolmogorov-Smirnov. O teste KS é adequado numa situação em que os parâmetros da distribuição hipotética são completamente conhecidos. No entanto, por vezes, é difícil inicialmente ou completamente especificar os parâmetros quando a distribuição é desconhecida. Neste caso, os parâmetros precisam ser estimados com base nos dados da amostra.

Em contraste com o teste KS, os parâmetros para o Teste de Lilliefors são estimados com base na amostra. Portanto, nesta situação, o teste LF será preferido sobre o teste KS (RAZALI; WAH, 2011). Dada uma amostra de observações, a estatística LF é definida como apresentado na equação (3) (LILLIEFORS, 1967),

$D=\operatorname{má} x\left|F^{*}(x)-\operatorname{Sn}(X)\right|$

onde $S_{n}(x)$ é a função de distribuição acumulada da amostra e $F^{*}(x)$ é a função de distribuição normal acumulada com média $\mu=\bar{x}$ e variância $s^{2}$ definida com denominador $n-1$.

Aplicou-se o Teste Lilliefors para cada escore fatorial, e, para aqueles em que a normalidade não for comprovada, aplica-se as transformações sugeridas Johnson e Wichern (1998) que satisfaçam a condição de mudança de comportamento apresentada em Silva e Santos (2015). O quadro 3, apresenta os resultados obtidos após os testes estatísticos e as transformações aplicadas.

Analisando o quadro 3, observa-se que os escores fatoriais 4, 5 e 7 já possuíam a condição de normalidade, e para os escores 1 e 9 uma transformação foi realizada para garantir essa condição, nesses casos o Teste de Lilliefors aceita a hipótese nula e confirma a condição normalidade dos dados. Já os escores 2, 3, 6 e 8 não são normais e não se encontrou até o momento uma transformação para eles, logo os mesmos não serão utilizados no monitoramento da estrutura. No entanto, mais esforços serão destinados em trabalhos futuros com o objetivo de encontrar transformações para os escores não utilizados nesse artigo, e assim, os mesmos poderão ser incorporados e novos modos de falha monitorados. 
Quadro 3: Resultados obtidos após Teste Lilliefors

\begin{tabular}{|c|c|c|c|c|}
\hline Escores & Estimativas & Transformação & Estimativas & Decisão Teste \\
\hline 1 & $\mathrm{~d}=0.1209, \mathrm{p}<0.05$ & $\sqrt{\left|x_{i}\right|}$ & $\mathrm{d}=0.0520, \mathrm{p}>0.20$ & Normal \\
\hline 2 & $\mathrm{~d}=0.1470, \mathrm{p}<0.01$ & não & & \\
\hline 3 & $\mathrm{~d}=0.0713, \mathrm{p}<0.05$ & não & & Normal \\
\hline 4 & $\mathrm{~d}=0.0553, \mathrm{p}>0.20$ & - & & Normal \\
\hline 5 & $\mathrm{~d}=0.0037, \mathrm{p}>0.20$ & - & & Normal \\
\hline 6 & $\mathrm{~d}=0.0847, \mathrm{p}<0.01$ & não & & \\
\hline 7 & $\mathrm{~d}=0.0336, \mathrm{p}>0.20$ & - & & Normal \\
\hline 8 & $\mathrm{~d}=0.1246, \mathrm{p}<0.01$ & não & & \\
\hline 9 & $\mathrm{~d}=0.1491, \mathrm{p}<0.01$ & $\sqrt{\left|x_{i}\right|}$ & $\mathrm{d}=0.4487, \mathrm{p}>0.20$ & \\
\hline
\end{tabular}

\section{CORRELAÇÃO DOS FATORES COM AS ANOMALIAS}

De acordo com a análise fatorial realizada gerou-se grupos de instrumentos que são altamente correlacionados. $\mathrm{O}$ quadro 4 apresenta esses grupos.

Quadro 4: Grupos de instrumentos altamente correlacionados

\begin{tabular}{|c|c|c|l|l|}
\hline \multicolumn{5}{|c|}{ GRUPOS GERADOS } \\
\hline FATOR 1 & FATOR 4 & FATOR 5 & FATOR 7 & FATOR 9 \\
\hline Pêndulos & R. D 5/tr 1 & RD 6/tr4 & RD 4/tr1 & Piezômetro 41 \\
Termômetros & R. D 5/tr 2 & RD 6/tr5 & RD 4/tr2 & Piezômetro 43 \\
Tensômetros & R. D 5/tr 3 & RD 6/tr1 & RD 4/tr3 & Piezômetro 45 \\
JS-A e JS-D & R. D 5/tr 4 & & RD 4/tr4 & \\
Temp. ambiente & R. D 5/tr 5 & & & \\
Piezômetro 46 & & & & \\
\hline
\end{tabular}

Onde RD são as rosetas de deformímetros instalados em determinadas regiões do bloco A-15 e, JS-A, JS-D são as bases de alongâmetro instalados nos blocos A-15 e D-14, respectivamente. Alguns instrumentos apresentados no quadro 1, não foram agrupados nesses fatores, e como os demais fatores não dão origem a escores fatoriais com distribuição de probabilidade da normal, eles não entraram na determinação dos modos de falha e consequentemente na determinação de regiões críticas.

De acordo com o apresentado na literatura e o conhecimento prático dos profissionais em segurança de barragens de Itaipu é possível com esses grupos monitorar as seguintes anomalias: escorregamentos e subpressão, fluência e tombamento.

\subsection{Escorregamentos e Supressão}

O escorregamento de um bloco de uma barragem de concreto é quando há um movimento de abertura e deslizamento entre os blocos vizinhos, fazendo com que ele se movimente para uma determinada direção, no caso do bloco chave em estudo esse movimento pode ocorrer na direção vertical oposta ao reservatório, quando as bases de alongâmetro que estão altamente correlacionados com o fator 1 apresentam resultados fora de seu estado normal de comportamento. 
A subpressão é um efeito causado pela pressão exercida pela agua que percorre a fundação, aliviando o peso da barragem, contribuindo para o escorregamento da estrutura em uma determinada camada da fundação.

Como os escores fatoriais 1 e 9 após a transformação assumem uma distribuição normal, pode-se monitorar dois tipos diferentes de escorregamento, o escorregamento na junta $D$ e o escorregamento na brecha $D$. O escorregamento na junta $D$ pode ocorrer quando existe uma mudança no comportamento das bases de alongâmetro instaladas no bloco em conjunto com uma subpressão detectada pelos piezômetros instalados na junta D. Do mesmo modo, um escorregamento na brecha D pode ocorrer, com uma mudança de comportamento das bases de alongâmetro em conjunto com uma subpressão detectada pelos piezômetros instalados na brecha D.

A subpressão na brecha $D$ é quando os piezômetros instalados na Brecha $D$ que se encontra a uma determinada profundidade da fundação do bloco apresentam valores acima dos seus respectivos valores limites. Esses instrumentos estão associados ao fator 1 e 9.

\subsection{Fluência}

O comportamento reológico do concreto, isto é, sua deformabilidade dependente do tempo, tem uma considerável importância na análise estrutural. As deformações dependentes do tempo, são convencionalmente separadas em duas: a fluência e a retração. A fluência é o acréscimo contínuo das deformações que ocorre mesmo para uma tensão constante. A retração é a redução de volume do material na ausência de uma carga externa. Tanto a fluência, quanto a retração, diminuem com a redução do fator água-cimento e do consumo de cimento (ARAÚJO, 2002).

Os instrumentos capazes de monitorar uma fluência são as rosetas de deformímetro que estão altamente correlacionadas com os fatores 4, 5 e 7.

\subsection{Tombamento}

O tombamento, pode acontecer na direção de montante ou da jusante, como resultado das forças atuantes na estrutura. O tombamento é o mecanismo de ruptura da barragem como um todo, tendendo a fazê-la girar em torno de um ponto $A$. O tombamento é, no entanto, um mecanismo raro de ocorrer, o fato é que antes da ruptura por tombamento, desenvolvem-se esforços de tração e aumentos de sub-pressão na rocha à montante e aumentos das tensões de compressão à jusante, e com a existências das forças horizontais o resultado é a ruptura por deslizamento antes da ocorrência do tombamento (GUSMÃO FILHO, 2006).

O tombamento é um movimento quase que total da estrutura, e por esse motivo praticamente todos os instrumentos instalados são afetados quando essa anomalia ocorrer. Portanto, nesse caso, todos os fatores apresentados no quadro 3 podem contribuir para identificação de um tombamento.

Para gerar o modelo para o caso aplicado é conveniente apresentar a associação dos fatores com as anomalias, o quadro 5 a seguir enumera cada anomalia e apresenta essa associação: 
Quadro 5 - Associação dos fatores com as anomalias

\begin{tabular}{|c|l|l|}
\hline Índice & \multicolumn{1}{|c|}{ Anomalia } & \multicolumn{1}{|c|}{ Fatores Envolvidos } \\
\hline 1 & Escorregamento na Brecha D & 1,9 \\
2 & Subpressão na Brecha D & 9 \\
3 & Escorregamento na Junta D & 1 \\
4 & Fluência & $4,5,7$ \\
5 & Tombamento & $1,4,5,7,9$ \\
\hline
\end{tabular}

\section{REGIÕES DE FALHA PARA O CASO APLICADO}

De acordo com o apresentado nesse artigo pode-se monitorar as anomalias apresentadas no quadro 4. Para determinar as regiões críticas dessas anomalias utiliza-se o algoritmo representado pelo fluxograma apresentado na Figura 2. Para determinar cada região de falha fazse simulações, onde apenas os instrumentos relacionados com uma determinada anomalia apresente valores dentro dos intervalos $M C$, utilizando para isso apenas os fatores que possuem influência para aquela anomalia, determinado a região crítica correspondente.

Por exemplo, para gerar a região crítica da primeira anomalia apresentado no Quadro 4, realiza-se simulações com os instrumentos relacionados ao escorregamento na brecha $D$, gerando valores para os escores fatoriais 1 e 9, obtendo as regiões críticas $R C_{11}$ e $R C_{19}$, correspondentes as regiões críticas da anomalia 1 a partir do fator 1 e região crítica da anomalia 1 a partir do fator 9 .

Os intervalos de projeto utilizados para gerar os intervalos de mudança de comportamento para casa instrumento foram cedidos pela Itaipu e constam nos relatórios Itaipu (1995) e Itaipu (1984). Os valores utilizados para as constantes $k_{1}$ e $k_{2}$, que assumem papel fundamental para a geração desses intervalos, assumiram valores iguais a dois, ou seja, para garantir que os vetores realmente simulassem uma mudança de comportamento, foi considerado um deslocamento de dois desvios padrões, no intervalo de confiança de $95 \%$ da média.

Dessa forma obtiveram-se após 2500 simulações as regiões críticas para as anomalias citadas no Quadro 4.
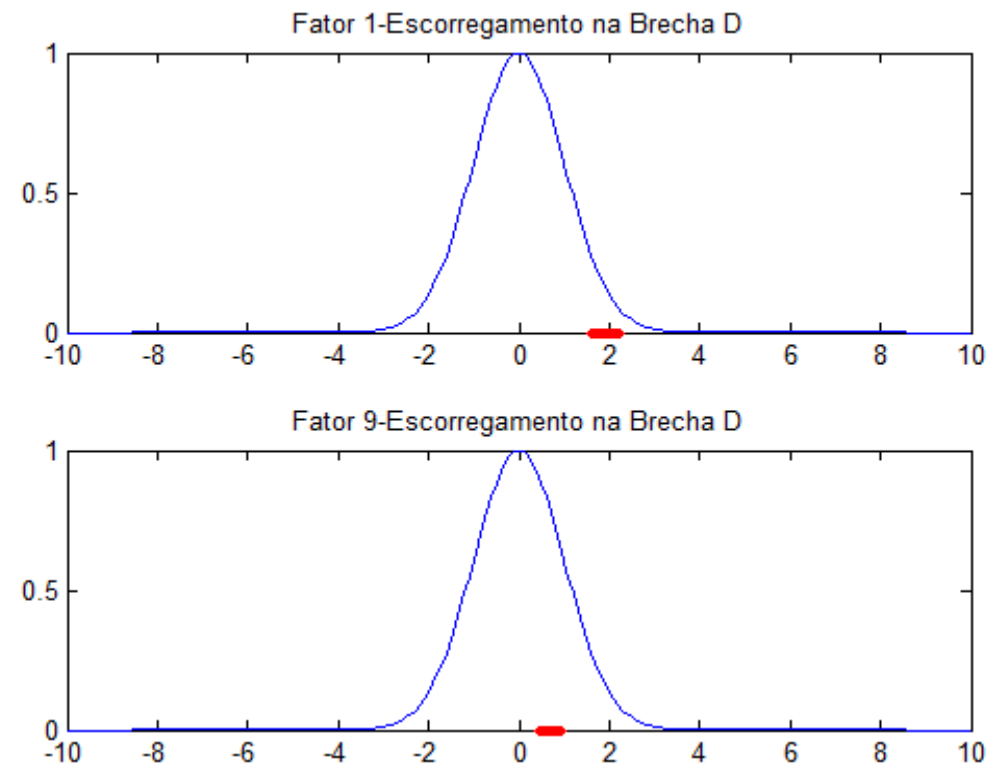
Figura 5: Escorregamento Brecha D

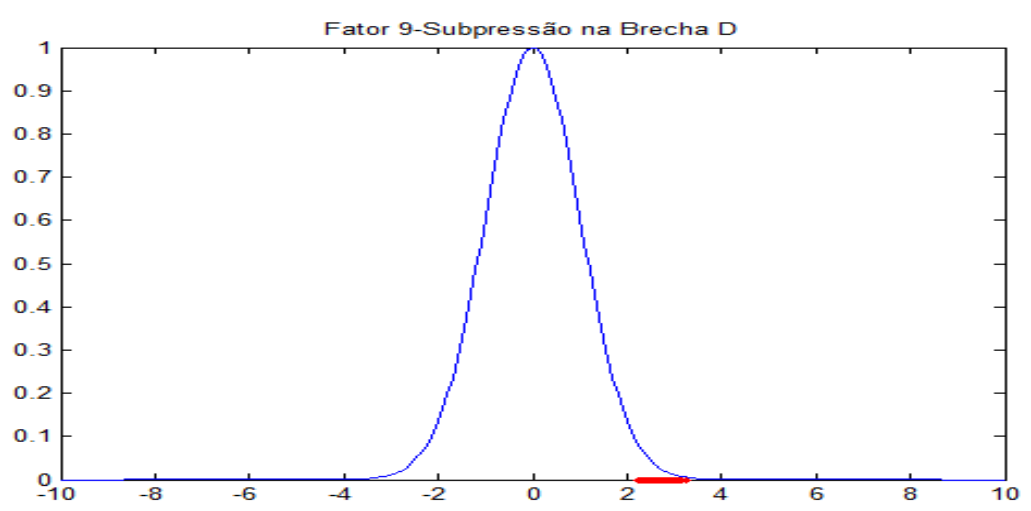

Figura 6: Subpressão na Brecha D

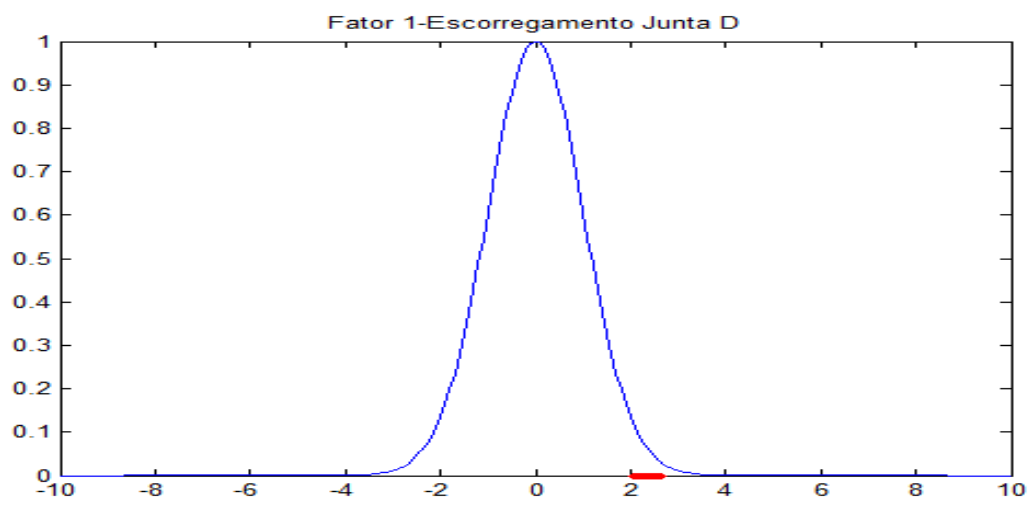

Figura 7: Escorregamento Junta D

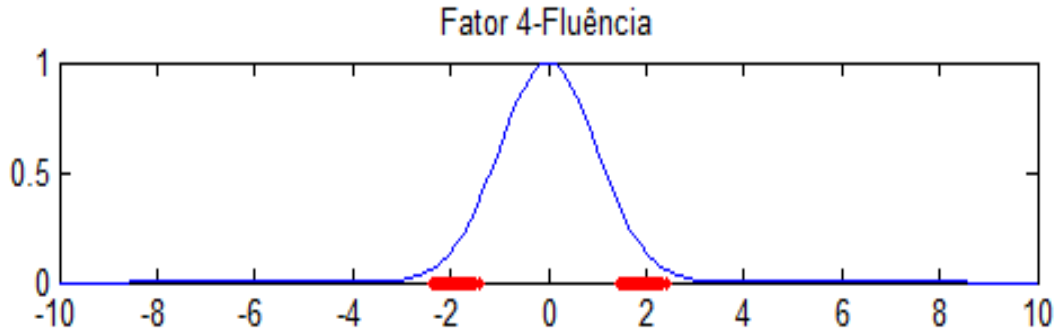

Fator 5-Fluência

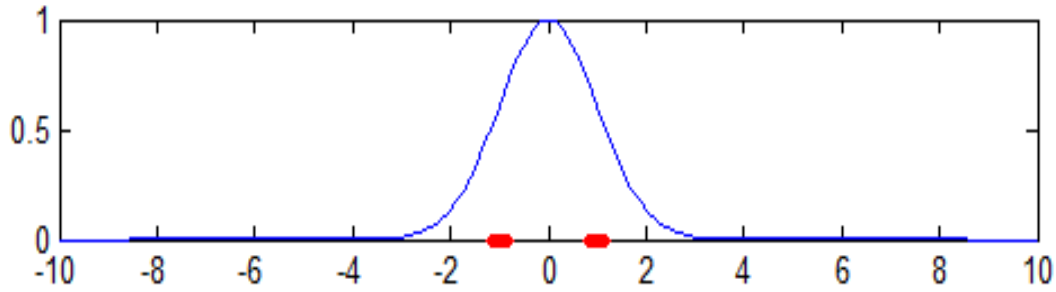

Fator 7-Fluência

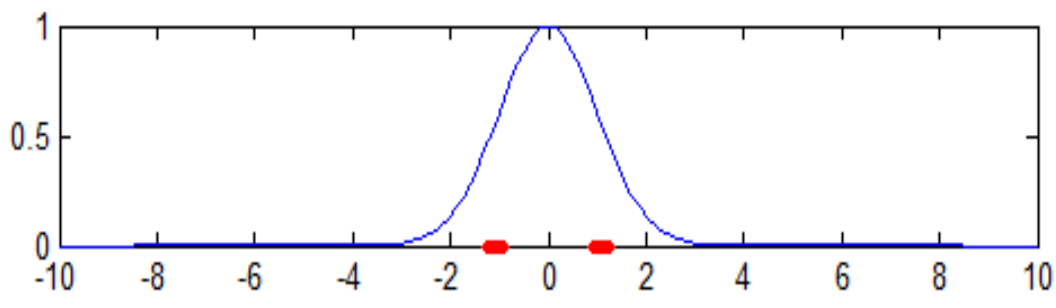

Figura 8: Fluência 
Fator 1-Tombamento
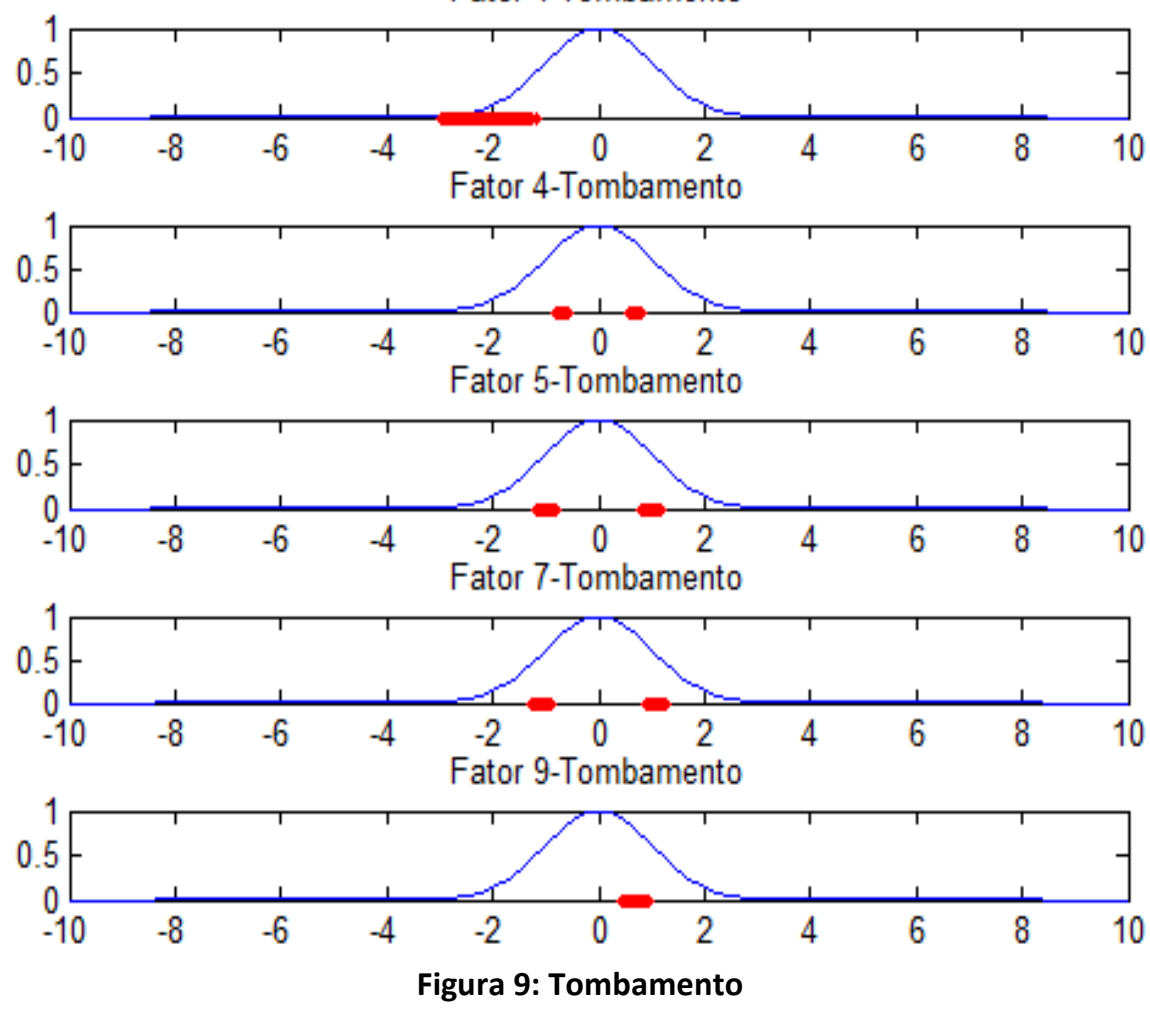

\section{RESULTADOS E DISCUSSÃO}

Foi aplicado o método proposto por Silva e Marques (2015) no bloco chave A-15 a Usina Hidrelétrica de Itaipu. A aplicação nesse bloco foi possível graças à confirmação de todos os testes estatísticos disponíveis na literatura, que dão respaldo teórico para aplicação de técnicas da Análise Multivariada.

Para aplicação da análise fatorial foi utilizado uma matriz gerada das leituras dos instrumentos instalados no bloco A-15 da barragem, correspondente aos últimos 13 anos, que estão disponíveis em um banco de dados, que foi fornecido pelos profissionais em segurança de barragens de Itaipu.

Para obtenção de regiões críticas de anomalias como, escorregamento, subpressões, fluência e tombamento foram utilizados dados sintéticos que simulavam a ocorrência de uma determinada anomalia. A obtenção desses dados foi possível graças à utilização do algoritmo proposto por Silva e Marques (2015) e representado pelo fluxograma apresentado na figura 2.

Os resultados obtidos corroboraram com a tese de que quando os escores fatoriais são gerados por vetores que simulam uma determinada anomalia, os valores dos escores tende a se afastar da média zero, desde que os escores fatoriais sejam variáveis aleatórias com distribuição de probabilidade normal.

Verificou-se que existe a possibilidade de aumentar o número de anomalias possíveis de ser monitoradas, desde que se encontrem funções capazes de tornar os escores fatoriais que não puderam ser transformados em variáveis aleatórias normais. No entanto, mesmo para os casos onde nem todos os escores possuíam a condição de normalidade, o método apresentado 
permite um monitoramento mais rápido da ocorrência de algumas dessas anomalias. Para tanto, basta que ao ser coletado leituras de todos instrumentos instalados no bloco em um determinado mês, utilize-se as informações obtidas nesse artigo e calcule-se a partir desse vetor mensal, o valor dos respectivos escores fatoriais, plotando esses pontos sobre as respectivas regiões críticas determinadas. Se ocorrer a combinação que indica uma determinada anomalia, pode-se dizer que existe indícios estatísticos para acreditar que tal fenômeno pode estar ocorrendo, ou na eminência de ocorrer, auxiliando na tomada de decisão.

\section{CONCLUSÃO}

O método proposto é uma alternativa para o monitoramento de anomalias em um bloco de concreto altamente instrumentado. Por ser um método estatístico, sua eficiência depende da qualidade da coleta e padronização dos dados e da comprovação de algumas hipóteses, que essencialmente são a confirmação de todos os testes estatísticos necessários para aplicação de técnicas de análise multivariada, bem como, da condição de normalidade dos escores fatoriais, que podem algumas vezes ser obtida através de transformações nos dados.

Para melhoria do método pretende-se aperfeiçoar a coleta e padronização dos dados, aplicando um planejamento estratégico tático, possibilitando a coleta dos dados em datas e horários mais próximos possíveis. Com esse planejamento pretende-se diminuir os erros de aproximações com as interpolações, e erros de correlações nos dados, já que a temperatura é uma variável que influencia praticamente todos os instrumentos.

O planejamento tático a ser formulado permitirá uma coleta mais robusta dos dados, e, em conjunto com a análise estatística mencionada, um monitoramento mais eficiente pode ser obtido, o qual poderá ser repetido para os demais blocos chave.

Para melhor entendimento dos resultados obtidos nesse trabalho, que são essencialmente as regiões críticas para cada modo de falha apresentadas no quadro 5, assume-se cada modo de falha como um evento em paralelo, onde a condição para que cada falha ocorra seja uma determinada combinação de resultados para os escores fatoriais obtidos, a partir de um vetor com as médias das leituras mensais de todos instrumentos instalados no bloco em estudo. Trabalhos futuros serão realizados para que mais anomalias possam ser detectadas, apresentando outras transformações que possibilitem a normalização dos escores fatoriais, e, para uma representação adequada desses fenômenos através de uma árvore de eventos.

\section{REFERÊNCIAS}

1. ICOLD. Critérios de Projeto Civil de Usinas Hidrelétricas. Centrais Elétricas Brasileiras S.A. ELETROBRÁS. 2003.

2. Agência Nacional de Águas (ANA). Conjuntura de Recursos Hídricos no Brasil. 432-f. Ministério do Meio Ambiente. Brasília, 2013.

3. Brasil. Lei $n^{\circ} 12.334$, de 20 de setembro de 2010 .

4. Brasil. Resolução $n^{\circ} 144$ de 10 de julho de 2012.

5. FILHO, J. G. Desempenho de Obras Geotécnicas. 523p. Editora Universitária da UFPE. Recife, 2006. 
6. SILVEIRA, J. F. A. Instrumentação e Segurança de Barragens de Terra e Enrocamento. 208p. Editora Oficina de Textos, São Paulo, 2003.

7. ELETROBRÁS. Critérios de Projeto Civil de Usinas Hidrelétricas. vol 1. Rio de Janeiro, 2003.

8. SILVA, O. C. ; MARQUES, J. M.. Estudo da Variabilidade dos Instrumentos Instalados em Blocos Chave da Barragem de Itaipu para Determinação de Modos de Falha. Em: XXX SNGB Seminário Nacional de Grandes Barragens, Maio, 10-12. Comitê Brasileiro de Grandes Barragens - CBDB, Foz do Iguaçu, 2015.

9. JOHNSON, R. A.; WICHERN, D. W. Applied Multivariate Statistical Analysis. Fourth Edition. Prentice Hall . New Jersey, 1998.

10. CORRAR, L. J.; PAULO, E. ;FILHO, J. M. Análise Multivariada para os Cursos de Administração, Ciências Contábeis e Economia. 539-f (73-129). Atlas. São Paulo, 2009.

11. SILVA, O. C.; SANTOS, C. G.; et al. Otimização da Análise de Variabilidade de Blocos Chaves da Barragem de Itaipu com o uso de novas Estratégias na coleta de dados e Técnicas para Normalização dos Escores Fatoriais. Em: Congresso de Métodos Numéricos em Engenharia, Lisboa, 29 de Junho à 2 de Julho. APMTAC. Portugal, 2015.

12. ARAÚJO, J. M. de. Estruturas de concreto : modelos de previsão da fluência e da retração do concreto. $n^{\circ} 4$. Rio Grande. Dunas, 2002.

13. ITAIPU. Usina Hidrelétrica de Itaipu - Aspectos Teóricos de Engenharia. 784-f (01-784). TAB Marketing Editorial. Foz do Iguaçu, 2003.

14. BIASE, A. G.; FERREIRA, D. F. Teste Computacionalmente Intensivo Baseado na Distância de Mahalanobis para Normalidade Multivariada. 22-f (1 - 22). Bras. Biom, Universidade Federal de Lavras, V. 30, N 1. 2012.

15. RAZALI, N. M.; WAH, Y. B. Power comparisons of shapiro-wilk, kolmogorov-smirnov, lilliefors and anderson-darling tests. Journal of Statistical Modeling and Analytics, v. 2, n. 1, p. $21-33$, 2011.

16. LILLIEFORS, H. W. On the Kolmogorov-smirnov Test for Normality with Mean and Variance Unknown. 3-f (399 - 402). Journal of the American Statistical Association, V. 62. N³18. 1967.

17. ITAIPU. Relatório do Histórico do Desempenho das Estruturas e suas Fundações. Aproveitamento Hidrelétrico de Itaipu. 533-f. Relatório N 40065000024P(1)ROA. IECO-ILC. Curitiba, 1995.

18. ITAIPU. Barragem Lateral Direita e Vertedouro Instrumentação - Previsão do Comportamento. Aproveitamento Hidrelétrico de Itaipu. 67-f. Relatório N 4066-50-5000-PR2.ECO. Foz do Iguaçu, 1984.

19. ITAIPU. Usina Hidrelétrica de Itaipu. Sistema e Operação e Manutenção de Funcionamento. Descrição de Funcionamento - DDF.Cl.025, DDF.Cl.006, DDF.Cl.015, DDF.Cl.016, DDF.Cl.019, DDF.Cl.020 - R00. Conjunto de Obras Civis. Julho, 2007. 